\title{
RECUERDO DEL PROF. DR. RUBÉN O. DE MARCO
}

\author{
MEMORY OF PROF. DR. RUBÉN O. DE MARCO
}

Con Rubén nos conocimos en el año 1966, cuando él era ayudante alumno de la Cátedra de Farmacología de la Facultad de Ciencias Médicas de La Plata, cuyo Profesor era el Dr. Manuel Litter, y yo alumno del tercer año de la carrera. Años después compartimos guardias como médicos de reciente graduación en distintas oportunidades.

Nació el 19 de enero de 1942 en la Ciudad de La Plata donde cursó sus estudios primarios y secundarios. En 1967 se graduó como Doctor en Medicina y a partir de allí comenzó una brillante carrera como médico asistencial y docente. Al comienzo se inclinó por la Especialidad de Medicina Interna y se desempeñó como médico de la Sala IV de HIGA San Martín de La Plata hasta el año 1973. En ese mismo año fue designado Jefe de la Unidad Sanitaria NN 16 de Berisso por concurso de Carrera Médico Hospitalaria. En 1994 fue nombrado Jefe del Servicio de Clínica Médica del Hospital Zonal de Berazategui "Evita Pueblo" hasta el año 2000, lugar donde ejerció también la Jefatura del Área de Docencia e Investigación.

Transcurría el año 2000 cuando comenzó su actividad en el Hospital de Alta Complejidad en Red El Cruce como Jefe del Área de Docencia e Investigación. Cabe señalar que a mediados de los años 1970 se despertó su otra gran pasión: la Diabetología, y la atención de su consultorio particular se centró prácticamente en pacientes que padecían este trastorno metabólico.

Fue Presidente de la Sociedad Médica de La Plata, fundador y posterior Presidente de la Sociedad de Medicina Interna de La Plata y subdirector del Hospital Español de La Plata.
Su actividad como Docente en Medicina Interna fue por demás exitosa, ya que ocupó diferentes cargos para finalizar como Profesor Titular Semidedicación de la Cátedra de Medicina Interna "B" de la Facultad de Ciencias Medicas de La Plata, cargo que obtuvo por concurso de oposición y antecedentes en el año 2005. Entre sus otros logros se destacan:

- Estuvo a cargo de la Cátedra de Postgrado para el Título en Clínica Médica desde 1983 a 1985.

- Fue Director del Curso Bianual para optar por el Título de Especialista en Clínica Médica Hospital de Berazategui, Distrito II desde 1995 hasta 2005.

- Publicó numerosos trabajos científicos de Medicina Interna y en los últimos 20 años exclusivamente de Diabetología en revistas nacionales y extranjeras.

- Fue coautor en diversos libros sobre temas de Diabetología, y su libro más conocido y entrañable "Diabetes mellitus clínica y tratamiento" en colaboración con el Prof. Dr. Jorge Salvioli, con edición agotada en Argentina.

- Se desempeñó como Director de múltiples cursos de Medicina Interna y Diabetología, como así también dictado de conferencia de esta última especialidad en Cursos, Jornadas, Mesas Redondas y Congresos Nacionales e Internacionales.

- Fue Vicepresidente del Comité Científico de la Sociedad Argentina de Diabetes (SAD) y del Congreso Anual de Diabetes en Mendoza (octubre de 2000).

- Trabajó como coordinador General del Comité Científico de la SAD (enero de 2001).

- Fue designado Presidente de la SAD en 
el año 2002 por el término de dos años donde pudo cumplir prácticamente todos los objetivos proyectados. Su gran ilusión era realizar el Congreso Argentino de Diabetes en la Ciudad de La Plata, pero la falta de complejidad hotelera fue un impedimento.

- Chairman; Bernardo Houssay Memorial Lecture (Homenaje a la Diabetes Argentina) en Congreso Mundial de Diabetes, París, 26 de agosto de 2003.

- Fue Presidente del Congreso Argentino de Diabetes en Mar del Plata (octubre de 2004).

Rubén también fue un creador nato de ideas y proyectos que ponía en ejecución en forma inmediata y permanente. Supo ganarse la admiración y el afecto de colegas, amigos y pacientes. Ayudaba a sus amigos, colaboradores y discípulos en todos los aspectos de la vida, fuesen científicos o de índole particular. Ofició de guía formativo a todos aquellos que lo rodearon. Fue un entusiasta sin límites, con gran valentía para defender sus convicciones. El espíritu docente y la dedicación hacia el paciente fueron sus características más destacadas.

Estar en el mismo Hospital y ser ambos docentes, además de compartir iguales ideales políticos universitarios determinaron una comunicación permanente que a través de los años se transformó en una sólida amistad. Es por ello que Rubén merece ser recordado no exclusivamente desde el punto de vista docente y profesional, sino también por ser abierto a quienes lo rodearon tratando siempre de hacerlos progresar. Falleció el 28 de mayo de 2017. 